\title{
Urate transport capacity of glucose transporter 9 and urate transporter 1 in cartilage chondrocytes
}

\author{
BINGQING ZHANG $^{1 *}$, MENGYUAN DUAN $^{2 *}$, BO LONG $^{3}$, BAOZHONG ZHANG $^{4}$, DONGMEI WANG ${ }^{5}$,

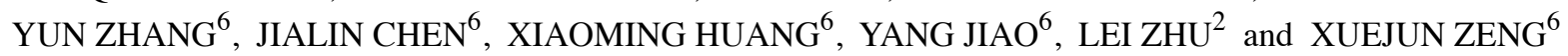

${ }^{1}$ Department of Internal Medicine, Peking Union Medical College Hospital, Chinese Academy of Medical Science and Peking Union Medical College, Beijing 100730; ${ }^{2}$ Department of Pharmacology, Institute of Basic Medical Sciences, Chinese Academy of Medical Sciences and School of Basic Medicine, Peking Union Medical College, Beijing 100005;

${ }^{3}$ Central Research Laboratory; ${ }^{4}$ Department of Orthopedics, Peking Union Medical College Hospital, Chinese Academy of Medical Sciences and Peking Union Medical College, Beijing 100730; ${ }^{5}$ Department of Neurology, Southern Medical University Nanfang Hospital, Guangzhou, Guangdong 510515; ${ }^{6}$ Department of General Medicine, Peking Union Medical College Hospital, Chinese Academy of Medical Science and Peking Union Medical College, Beijing 100730, P.R. China

Received November 22, 2018; Accepted May 31, 2019

DOI: $10.3892 / \mathrm{mmr} .2019 .10426$

\begin{abstract}
Chronic gouty arthritis, caused by a persistent increase in, and the deposition of, soluble uric acid (sUA), can induce pathological chondrocyte destruction; however, the effects of urate transport and intracellular sUA on chondrocyte functionality and viability are yet to be fully determined. Thus, the aim of the present study was to investigate the presence and functionality of a urate transport system in chondrocytes. The expression profiles of two primary urate reabsorptive transporters, glucose transporter 9 (GLUT9) and urate transporter 1 (URAT1), in human articular cartilage and chondrocyte cell lines were examined via western blotting, reverse transcription-quantitative PCR, immunohistochemistry and immunofluorescence. Then, chondrocytes were incubated with exogenous sUA at increasing concentrations. Negative control assays were conducted via the specific knockdown of GLUT9 and URAT1 with lentiviral short hairpin (sh)RNAs, and by pretreatment with benzbromarone,
\end{abstract}

Correspondence to: Dr Xuejun Zeng, Department of General Medicine, Peking Union Medical College Hospital, Chinese Academy of Medical Science and Peking Union Medical College, 1 Shuaifuyuan Street, Dongcheng, Beijing 100730, P.R. China E-mail: zxjpumch@126.com

Dr Lei Zhu, Department of Pharmacology, Institute of Basic Medical Sciences, Chinese Academy of Medical Sciences and School of Basic Medicine, Peking Union Medical College, 5 Dongdansantiao Street, Dongcheng, Beijing 100005, P.R. China

E-mail: leizhu2004@126.com

${ }^{*}$ Contributed equally

Key words: gouty arthritis, chondrocyte, urate transporter, uric acid, benzbromarone a known inhibitor of the two transporters. Intracellular UA concentrations were measured using colorimetric assays. The expression levels of GLUT9 and URAT1 were determined in cartilage tissues and chondrocyte cell lines. Incubation of chondrocytes with sUA led to a concentration-dependent increase in intracellular urate concentrations, which was inhibited by GLUT9 or URAT1 knockdown, or by benzbromarone pretreatment $(27.13 \pm 2.70,44.22 \pm 2.34$ and $58.46 \pm 2.32 \%$ reduction, respectively). In particular, benzbromarone further decreased the already-reduced intracellular UA concentrations in HC-shGLUT9 and HC-shURAT1 cells by $46.79 \pm 2.46$ and $39.79 \pm 2.22 \%$, respectively. Cells overexpressing GLUT9 and URAT1 were used as the positive cell control, which showed increased intracellular UA concentrations that could be reversed by treatment with benzbromarone. In conclusion, chondrocytes may possess an active UA transport system. GLUT9 and URAT1 functioned synergistically to transport UA into the chondrocyte cytoplasm, which was inhibited by specific gene knockdowns and drug-induced inhibition. These results may be fundamental in the further investigation of the pathological changes to chondrocytes induced by sUA during gouty arthritis, and identified UA transport processes as potential targets for the early control of chronic gouty arthritis.

\section{Introduction}

Chronic gouty arthritis is a crystal-forming type of arthritis that causes severe inflammation and irreversible bone erosion, in which chondrocytes may serve an important role. Patients with gout display severe cartilage destruction and empty chondrocyte lacunae upon histopathological examination (1). A number of studies have demonstrated reduced cell viability and impaired functionality of chondrocytes following stimulation with monosodium urate monohydrate (MSU) crystals (1-3); however, MSU is precipitated from synovial soluble uric acid (sUA) in vivo only under certain conditions (4). Thus, the impact of sUA on chondrocytes may occur prior to MSU 
formation in gout, which is characterized by hyperuricemia. It was reported that sUA could lead to cellular dysfunction and a loss of viability in numerous cell types, including renal tubular cells (5), endothelial cells (6), vascular smooth muscle cells $(7,8)$, hepatocytes $(9)$, pancreatic $\beta$-cells $(10,11)$ and adipocytes (12); however, at present, few studies have focused on the effects of high levels of sUA on chondrocytes.

The oxidative properties of sUA require entry into the intracellular environment; previous experiments have demonstrated that inhibiting the entry of sUA into the cytoplasm blocked sUA-induced oxidative damage (5-9,12-14). Glucose transporter 9 (GLUT9) and urate transporter 1 (URAT1), the most notable reabsorptive urate transporters, regulate the access of sUA into the cytoplasm under normal and pathological conditions (15). GLUT9 serves a major role in UA homeostasis via its dual role in UA handling in the kidneys and uptake in the liver (16). In the Chinese population, a missense mutation in the GLUT9 gene was associated with chronic gouty arthritis (17); however, its UA transport capacity in chondrocytes and further actions in gout-associated cartilage damage are yet to be determined. URAT1 has been reported to be expressed in renal tubular cells (18), pancreatic $\beta$-cells $(10,11)$, endothelial cells (6), vascular smooth muscle cells (19) and adipocytes (12). The expression and uric transport function of URAT1 in chondrocytes have not been investigated.

To the best of our knowledge, whether there is a UA transport process in chondrocytes has not been previously studied, and the contribution of UA transport in chondrocytes to gouty arthritis is yet to be identified. Therefore, the expression profiles of GLUT9 and URAT1, and their contribution to UA transport capacity in chondrocytes were investigated in the present study.

\section{Materials and methods}

Tissue samples. The present study was approved by the Institutional Review Board of Peking Union Medical College Hospital (permit no. ZS-1445), and was conducted in accordance with the Declaration of Helsinki. Human articular cartilage (AC) was obtained from patients at the Orthopedic Department of the Peking Union Medical College Hospital between December 2017 and March 2018. All patients signed informed consent prior to the collection of samples. The inclusion criteria were a diagnosis of femoral neck fracture due to trauma and a requirement for a total hip replacement. Patients were excluded if they exhibited: i) Hyperuricemia; ii) gout; iii) arthritis; iv) rheumatoid arthritis; v) femoral head necrosis of any origin; and vi) cancer. AC samples were obtained from the otherwise discarded femoral head. AC tissue was immediately snap-frozen in liquid nitrogen, with the remaining sample immersed in $10 \%$ formalin at room temperature overnight for paraffin embedding. The clinical characteristics of the 5 patients from whom the AC samples were obtained are presented in Table SI.

Immunohistochemistry. Immunohistochemical assays were performed as previously described (20). Cartilage sections (thickness, $5 \mu \mathrm{m}$ ) were immersed at room temperature within $3 \%$ hydrogen peroxide in the dark for $25 \mathrm{~min}$ and then $3 \% \mathrm{BSA}$ (cat. no. A8010; Beijing Solarbio Science \& Technology Co., Ltd.) for $30 \mathrm{~min}$ at room temperature to block the endogenous peroxidase and non-specific protein, respectively. Then the sections were incubated at $4^{\circ} \mathrm{C}$ overnight with rabbit polyclonal antibodies against collagen II (1:100; cat. no. ab34712; Abcam), human GLUT9 (1:100; cat. no. ab223470; Abcam), and human URAT1 (1:100; cat. no. 14937-1-AP; ProteinTech Group, Inc.). PBS was used as an negative control (Fig. S1). Sections were labeled with horseradish peroxidase (HRP)-conjugated goat anti-rabbit immunoglobulin $\mathrm{G}$ heavy and light chain (IgG H\&L) secondary antibody $(1: 1,000$; cat. no. ab6721; Abcam) at room temperature for $50 \mathrm{~min}$. Resolution with 3,3'-diaminobenzidine substrate (cat. no. DA1010; Beijing Solarbio Science \& Technology Co., Ltd.) was guided microscopically. Cell nuclei were counterstained with aqueous hematoxylin at room temperature for $3 \mathrm{~min}$. Digital images were captured using a Nikon Microphot-FX microscope (magnification x100 and x200; Nikon Corporation).

Human articular chondrocyte cell line and gene knockdown. The human articular chondrocyte (HC-a) cell line, derived from human ACs, was purchased from ScienCell Research Laboratories, Inc. (cat. no. 4650). Cells were cultured at $37^{\circ} \mathrm{C}$ in an humidified incubator with $5 \% \mathrm{CO}_{2}$ with complete chondrocyte medium that contained $5 \% \mathrm{FBS}, 1 \%$ chondrocyte growth supplement and $1 \%$ penicillin/streptomycin (cat. no. 4651; ScienCell Research Laboratories, Inc.).

Gene knockdown was performed as previously described (21). HC-a cells were cultured 24-well plates until they reached $1 \times 10^{6}$ cells/well, and the medium was replaced fresh medium containing $6 \mu \mathrm{g} / \mathrm{ml}$ Polybrene ${ }^{\circledR}$ (cat. no. sc-134220; Santa Cruz Biotechnology, Inc.). A total of $10 \mu \mathrm{l}$ lentiviral particles containing short hairpin (sh)RNA against SLC2A9 (the gene encoding GLUT9; cat. no. sc-105399-V; Santa Cruz Biotechnology, Inc.) or SLC22A12 (the gene encoding URAT1; cat. no. sc-96373-V; Santa Cruz Biotechnology, Inc.) were then added and incubated for at $37^{\circ} \mathrm{C} 24 \mathrm{~h}$. Scrambled vector (cat. no. sc-108080; Santa Cruz Biotechnology, Inc.) was used as a negative control. Cells were then cultured in fresh complete medium and allowed to grow to $90 \%$ confluency for $\sim 24 \mathrm{~h}$.

Humane embryonic kidney cells 293 and gene overexpression. 293 cells were purchased from the National Infrastructure of Cell Line Resource (cat. no. 3111C0001CCC000010). 293 cells were cultured at $37^{\circ} \mathrm{C}$ with $5 \% \mathrm{CO}_{2}$ in HyClone ${ }^{\mathrm{TM}}$ Minimal Essential Medium with Earle's (cat. no. SH30024.01; Beijing Solarbio Science \& Technology Co., Ltd.) supplemented with $10 \%$ FBS (cat. no. P30-3302; PAN-Biotech).

293 cells were cultured on 24 -well plates to a density of $1 \times 10^{6}$ cells/well and then subjected to gene overexpression as previously reported (22). The recombinant plasmids SLC2A9 pcDNA3.1-T2A-EGFP (Fig. S2) and SLC22A12 pcDNA3.1-T2A-EGFP (Fig. S3) were purchased from Hanbio Biotechnology Co., Ltd. Briefly, $0.8 \mu$ g plasmids were mixed with $2 \mu \mathrm{l}$ LipoFiter ${ }^{\mathrm{TM}}$ liposomal transfection reagent (car. no. HB-TRLF-1000; Hanbio Biotechnology Co., Ltd.) for $20 \mathrm{~min}$ at room temperature and then added to cells. Empty vector (pcDNA3.1-T2A-EGFP; Hanbio Biotechnology Co., Ltd.) was used as a negative control. Following transduction 
for $6 \mathrm{~h}$, the medium was replaced with complete medium. Cells were then allowed to grow to $90 \%$ confluency for $\sim 24 \mathrm{~h}$.

Reverse transcription-quantitative PCR (RT-qPCR). Total RNA was extracted with TRIzol ${ }^{\circledR}$ (cat. no. DP405-02; Tiangen Biotech Co., Ltd.) according to the manufacturer's protocols. After converting the RNA to cDNA using a PrimeScript ${ }^{\mathrm{TM}}$ RT kit (cat. no. RR047B; Takara Biotechnology Co., Ltd.), qPCR was conducted using Bio-Rad iQ5 and SYBR Green Master Mix (cat. no. ABI7500; Bio-Rad Laboratories, Inc.) under the following thermocycling conditions: $95^{\circ} \mathrm{C}$ for $30 \mathrm{sec}$, followed by 40 cycles of $95^{\circ} \mathrm{C}$ for $5 \mathrm{sec}$ and $60^{\circ} \mathrm{C}$ for $40 \mathrm{sec}$, and then $95^{\circ} \mathrm{C}$ for $10 \mathrm{sec}, 60^{\circ} \mathrm{C}$ for $60 \mathrm{sec}$ and $95^{\circ} \mathrm{C}$ for $15 \mathrm{sec}$ to obtain the melting curve; finally, samples were slowly heated to $99^{\circ} \mathrm{C}$. The relative gene expression was quantified with the $2^{-\Delta \Delta \mathrm{Cq}}$ method (23). Data were normalized to $\beta$-actin mRNA levels. The primers used were as follows: GLUT9, forward 5'-CCT GTTTGGAGTGATTGTGGT-3', reverse 5'CTTGCCTCG TTGTGCTTCTC-3'; URAT1, forward 5'GCCCGACACCAT CCAAGA-3', reverse 5'-TTCCTCTGACCGTCCCATC-3'; and $\beta$-actin, forward 5'CATCCCCCAAAGTTCACAAT3' and reverse 5'-AGTGGGGTGGCTTTTAGGAT-3'.

Immunofluorescence. Immunofluorescence staining was performed according to previously reported protocols (22). Cells were cultured on coverslips and fixed in $4 \%$ paraformaldehyde at room temperature for $15 \mathrm{~min}$. Following permeabilization with $0.1 \%$ Triton $\mathrm{X}-100$ and blocking with normal goat serum (cat. no. SL038; Beijing Solarbio Science \& Technology Co., Ltd.) at room temperature for 20 and $30 \mathrm{~min}$, respectively, cells were then incubated with rabbit polyclonal antibodies against human GLUT9 (1:100; cat. no. ab223470; Abcam) and human URAT1 (1:200; cat. no. 14937-1-AP; ProteinTech Group. Inc.) overnight at $4{ }^{\circ} \mathrm{C}$, followed by incubation with FITC-conjugated mouse anti-rabbit $\operatorname{IgG}(1: 1,000$; cat. no. sc-2359; Santa Cruz Biotechnology, Inc.) for $1 \mathrm{~h}$ at room temperature. PBS was used as a negative control (Fig. S4). Cell nuclei were counterstained with DAPI (cat. no. D21490; Invitrogen; Thermo Fisher Scientific, Inc.) at room temperature in the dark for $5 \mathrm{~min}$. Cells were imaged using a fluorescence photomicroscope (IX51; Olympus Corporation; magnification $\mathrm{x} 400)$ and analyzed with Image-Pro Plus 6.0 software (Media Cybernetics, Inc.) (24).

Protein preparation. Whole-cell protein lysates from cell lines and frozen tissue samples were prepared using RIPA lysis and extraction buffer (cat. no. 89901; Thermo Fisher Scientific, Inc.), and tissue extraction reagent I (cat. no. FNN0071; Thermo Fisher Scientific, Inc.) supplemented with Halt protease inhibitor cocktail (cat. no. 78425; Thermo Fisher Scientific, Inc.), respectively. To separate cell membrane and cytoplasmic proteins, a membrane and cytosol protein extraction kit (cat. no. P0033; Beyotime Institute of Biotechnology) was used. Briefly, cells were minced on ice and then centrifuged at $700 \mathrm{x} g$ for $10 \mathrm{~min}$ to remove cell nuclei and unminced cells. Samples were then centrifuged again at 14,000 x g for $30 \mathrm{~min}$ to collect the supernatant, which contained the cytoplasmic protein. The sediments were re-suspended with solution B from the kit, vortexed 2-3 times for $5 \mathrm{sec}$, and then further centrifuged at $14,000 \times \mathrm{g}$ for $5 \mathrm{~min}$, prior to collection of the final the supernatant, which contained the membrane protein. All centrifugations here were performed at $4^{\circ} \mathrm{C}$. Protein content was determined using a bicinchoninic acid kit (cat. no. P1511; Applygen Technologies, Inc.).

Western blot analysis. Western blotting was conducted as previously described (24). Proteins (30 $\mu \mathrm{g})$ were separated via $10 \%$ SDS-PAGE under reducing conditions, and transferred onto nitrocellulose membranes. Following blocking with $5 \%$ skimmed milk at room temperature for $1 \mathrm{~h}$, membranes were incubated with primary antibodies at $4^{\circ} \mathrm{C}$ overnight, and labeled with secondary antibodies at room temperature for $45 \mathrm{~min}$. The primary antibodies used for western blot analysis included: Rabbit polyclonal antibodies to collagen II (1:1,000; cat. no. ab34712; Abcam), human GLUT9 (1:1,000; cat. no. ab223470; Abcam), and human URAT1 (1:600; cat. no. 14937-1-AP; ProteinTech Group, Inc.). Mouse polyclonal antibodies against $\beta$-actin (1:1,000; cat. no. ab8226; Abcam), Na-K ATPase (EP1845Y; 1:5,000; cat. no. ab76020; Abcam) and GAPDH (EPR16891; 1:5,000; cat. no. ab181602; Abcam) were used as loading controls for whole cell, cell membrane and cytoplasmic protein extracts, respectively. PBS was used as a negative control (Fig. S5). HRP-conjugated goat anti-rabbit IgG H\&L (1:10,000; cat. no. ab6721; Abcam) and goat anti-mouse IgG H\&L (1:10,000; cat. no. ab205719; Abcam) antibodies were used as the secondary antibodies. Chemiluminescence was performed using Pierce ${ }^{\mathrm{TM}}$ Fast Western Blot ECL substrate (cat. no. 35055; Thermo Fisher Scientific, Inc.). Densitometric analysis of the band intensities was conducted using AlphaView Stand Alone (version 3.4.0, ProteinSimple) as previously described (24).

UA transport assays. Cells were cultured in 6-well plates to a density of $1 \times 10^{6}$ per well and washed with Krebs-Ringer bicarbonate (KRP) buffer (cat. no. G0430; Beijing Solarbio Science \& Technology Co., Ltd.). Cells were pretreated with KRP buffer containing $50 \mu \mathrm{M}$ benzbromarone for $30 \mathrm{~min}$ and incubated with $1 \mathrm{mM}$ benzbromarone for an additional 30 min. It was previously demonstrated that rapid UA transport occurs as early as 20 min post-incubation with UA (14), which could be inhibited by benzbromarone at a concentration of $50 \mu \mathrm{M}(25,26)$. Thus, for UA transport assays, cells were starved in KRP for $30 \mathrm{~min}$, followed by the addition of exogenous sUA (cat. no. U0881; Sigma-Aldrich; Merck KGaA) at concentrations of $0,0.25,0.50,0.75$ and $1 \mathrm{mM}$ at $37{ }^{\circ} \mathrm{C}$ for $30 \mathrm{~min}$. For inhibition assays, cells were pretreated with KRP buffer containing $50 \mu \mathrm{M}$ benzbromarone (J\&K Scientific Ltd.) at $37^{\circ} \mathrm{C}$ for $30 \mathrm{~min}$ and incubated with $1 \mathrm{mM} \mathrm{sUA}$ at $37^{\circ} \mathrm{C}$ for a further $30 \mathrm{~min}$.

Following this incubation, supernatants were removed, cells were washed twice with cold PBS and intracellular UA concentrations were measured using Colorimetric UA assay kits (cat. no. ab65344; Abcam) according to the manufacturer's protocols. Briefly, cells were resuspended in UA assay buffer and centrifuged at $13,000 \times \mathrm{g}$ at $4^{\circ} \mathrm{C}$ for $5 \mathrm{~min}$. Supernatants were collected and reaction solutions were added for $30 \mathrm{~min}$ at $37^{\circ} \mathrm{C}$ in the dark. The optical density at $570 \mathrm{~nm}$ was measured using a microplate reader. Standard curves were obtained with UA standards. 
Statistical analysis. Data were presented as the mean \pm standard deviation of at least three independent experiments, with the exception of western blot analysis of human cartilage samples, which presented protein for individual patients, with error bars indicating the variation observed in three replicates. A linear regression model was used to study the association between exogenous sUA and the intracellular UA concentration following incubation. Student's t-tests and one-way ANOVA followed by Bonferroni's test were used to compare results in two groups and $>2$ groups, respectively. $\mathrm{P}<0.05$ was considered to indicate a statistically significant difference. Stata14.0 (StataCorp LP) was used for statistical analysis.

\section{Results}

GLUT9 and URATI are expressed in human cartilage and $\mathrm{HC}$-a chondrocyte cells. A total of $5 \mathrm{AC}$ samples were collected during the present study. The clinical characteristics of the 5 patients from whom the AC samples were obtained are presented in Table SI. The expression levels of GLUT9 and URAT1 in the 5 human AC samples were examined by western blot and immunohistochemical analyses. Collagen II expression is a characteristic of chondrocytes (27), thus the detection of collagen II indicated the presence of chondrocytes in AC samples (Figs. 1 and 2). Western blot analysis using specific antibodies against GLUT9 and URAT1 revealed positive expression in all 5 samples (Fig. 1; Table SII). Immunohistochemical assays also revealed positive staining for GLUT9 and URAT1 in all AC samples (Fig. 2).

Furthermore, GLUT9 and URAT1 expression was observed in HC-a cells, the human articular chondrocyte cell line, via RT-qPCR (Fig. 3A), immunofluorescence (Fig. 3B) and western blot analyses (Fig. 3C; Tables SIII and SIV). Further separation of the membrane portion revealed high expression of the two transporters in the membrane protein extracts (Fig. 3D; Tables SIII and SIV), with limited expression in cytoplasmic protein extracts (Fig. 3E; Tables SIII and SIV).

HC-a cells display a concentration-dependent UA transport process. As the aforementioned results indicated the expression of two major urate transporters in chondrocytes, the present study then investigated the UA transport capacity of chondrocytes. sUA was added to HC-a cells at increasing concentrations $(0,0.25,0.50,0.75$ and $1 \mathrm{mM})$, and intracellular UA concentrations were measured using colorimetric UA assays. It was demonstrated that $\mathrm{HC}$-a cells displayed UA transport capacity, as evidenced by a concentration-dependent increase in intracellular UA, with a linear $\mathrm{R}^{2}$ of 0.993 (Fig. 4A; Table SV).

UA transport is inhibited by GLUT9 and URAT1 knockdown in $\mathrm{HC}$ - a cells. To study the contribution of GLUT9 and URAT1 to UA transport, $\mathrm{HC}$-a cells were transduced with lentiviral particles containing shRNAs to knockdown the expression of GLUT9 (HC-shGLUT9) and URAT1 (HC-shURAT1), with knockdown demonstrated via immunofluorescence, RT-qPCR and western blot analyses (Fig. 3). Then, HC-shGLUT9 and HC-shURAT1 cells were incubated with sUA $(1 \mathrm{mM})$ under the same conditions as the wildtype HC-a cells. Compared with wildtype cells, intracellular UA concentrations in
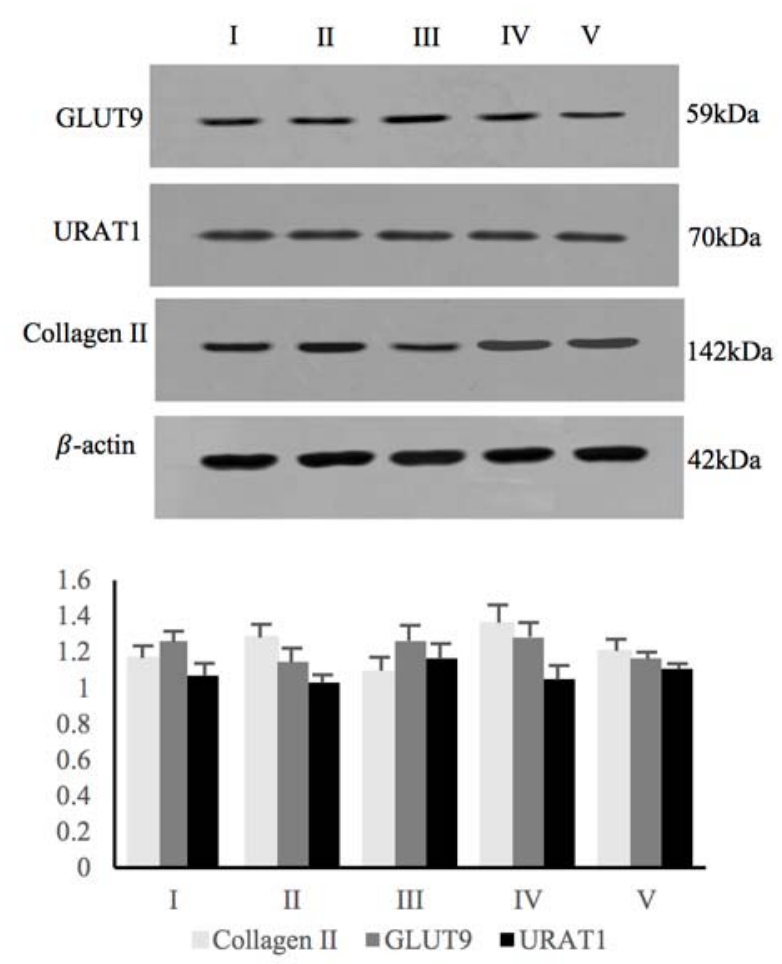

Figure 1. Protein expression of urate transporters in human cartilage. Human articular cartilage samples (I to V) exhibited positive expression of collagen II, GLUT9 and URAT1, as determined via western blot analysis. Error bars indicate western blot replicates. GLUT9, glucose transporter 9; URAT1, urate transporter 1.

HC-shGLUT9 and HC-shURAT1 cells were significantly decreased by $27.13 \pm 2.70$ and $44.22 \pm 2.34 \%$, respectively $(\mathrm{P}<0.05$; Fig. 4B; Table SVI). Cells transduced with scrambled vectors did not exhibit altered protein expression or UA transport capacity (Figs. 3 and 4; Tables SIII, SIV, and SVI). These data confirmed there may be a role for GLUT9 and URAT1 in the UA transport capacity of chondrocytes.

UA transport is inhibited by benzbromarone in HC-a cells. Previous studies have reported that $50 \mu \mathrm{M}$ benzbromarone, a widely used uricosuric agent, can reduce the UA transport capacity of GLUT9 and URAT1 by 68\% (25) and 93\% (26), respectively. Therefore, it was used to investigate the effects of the two Urate transporters in HC-a cells. The results revealed that benzbromarone treatment significantly reduced the intracellular UA concentration in HC-a cells by $58.46 \pm 2.32 \%$ $(\mathrm{P}<0.05$; Fig. 4B; Table SVI), but it did not affect protein expression or subcellular location in $\mathrm{HC}$-a cells, as determined by RT-qPCR and western blot assays (Fig. 3).

In HC-shGLUT9 and HC-shURAT1 cells, whose intracellular UA concentrations were already reduced following gene knockdown, benzbromarone pretreatment further decreased intracellular UA concentrations by $46.79 \pm 2.46$ and $39.79 \pm 2.22 \%$, respectively $(\mathrm{P}<0.05$; Fig. $4 \mathrm{~B}$; Table SVI), suggesting that GLUT9 and URAT1 acted synergistically to mediate UA transport in $\mathrm{HC}-\mathrm{a}$ cells.

UA transport is enhanced in 293 cells overexpressing GLUT9 and URAT1, which is reversed by benzbromarone treatment. 


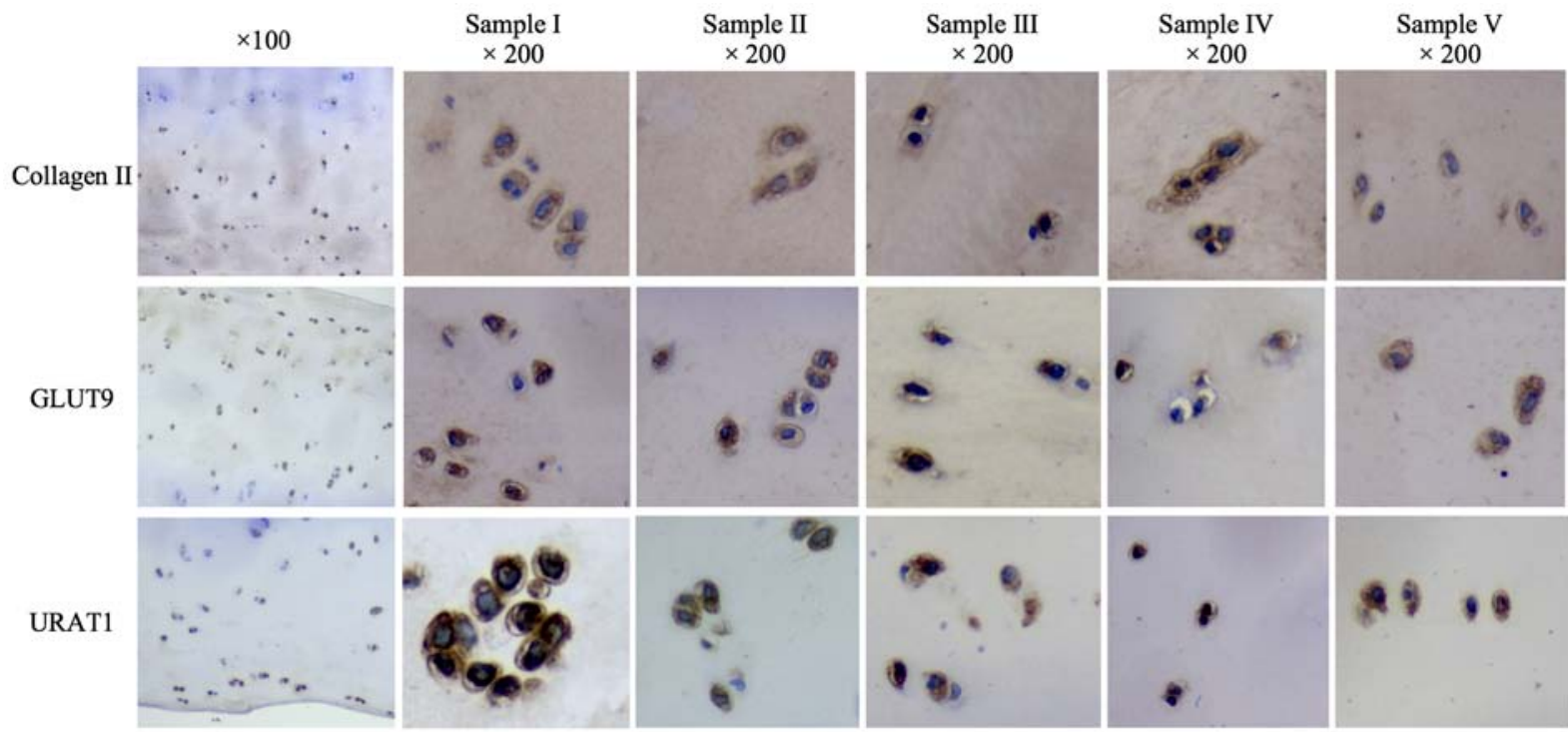

Figure 2. Histological analysis of urate transporter expression in human cartilage. Human articular cartilage samples (I to V) exhibited positive expression of collagen II, GLUT9 and URAT1, as determined via immunohistochemical analysis. Digital images were captured using a Nikon Microphot-FX microscope [magnification, x100 (Sample II) and x200 (all five samples)]. Representative images are shown. GLUT9, glucose transporter 9; URAT1, urate transporter 1.

To verify the sensitivity of the colorimetric UA kit and as a positive cell control, GLUT9 and URAT1 were overexpressed in 293 cells (Fig. 5A; Table SVII), a well characterized UA transport assay system $(14,28)$. Wildtype 293, GLUT9-293 and URAT1-293 cells were incubated with sUA $(0,0.25,0.50,0.75$, and $1 \mathrm{mM})$, and the intracellular UA levels were assessed. The results revealed enhanced UA transport with increasing concentrations of sUA in all 3 types of cell $\left(\mathrm{R}^{2}=0.954\right.$ for wildtype cells, 0.955 for GLUT9-293 cells and 0.947 for URAT1-293 cells). In addition, the intracellular UA concentrations were significantly increased in GLUT9-293 and URAT1-293 cells compared with the wildtype 293 cells at all sUA concentrations assessed (Fig. 5B; Table SVIII); however, pretreatment with benzbromarone significantly reversed the increased intracellular UA concentrations induced by the overexpression of GLUT9 and URAT1 (Fig. 5C; Table SIX). Cells transduced with empty vector did not exhibit altered protein expression or UA transport capacity (Fig. 5; Tables SVII and SIX). These findings were consistent with those of previous studies $(14,28)$ and thus demonstrated the sensitivity of the colorimetric UA transport assay.

\section{Discussion}

In the present study, the expressions of GLUT9 and URAT1 were demonstrated in AC tissues obtained from human cartilage via immunohistochemistry and western blot analyses, and then further verified using a human cartilage chondrocyte cell line (HC-a). The two proteins were predominantly expressed in the cell membrane, with limited expression in the cytoplasm. Functional studies revealed that wildtype HC-a cells exhibited a concentration-dependent increase in intracellular UA concentrations following incubation with exogenous sUA. Specific gene knockdown or functional inhibition of GLUT9 and URAT1 significantly decreased intracellular sUA. Therefore, to the best of our knowledge, the present study demonstrated for the first time that there is an active UA transport system in chondrocytes, which possessed two major urate transporters, GLUT9 and URAT1.

The confirmation of a UA transport system in chondrocytes is notable, as sUA turns into a strong pro-oxidative agent once transported into cytoplasm, and inhibiting this transport may prevent sUA-induced cell damage (5-9,12-14). Previous studies in other cell types revealed that intracellular sUA directly activates NADPH oxidase, which in turn actives the production of reactive oxygen species (ROS) (29). Increased ROS levels in turn stimulate protein kinases (MAPKs, ERKp44/42 and p38), proliferative factors (platelet-derived growth factor and transforming growth factor $\beta$ ) and proinflammatory transcription factors $(\mathrm{NF}-\kappa \mathrm{B})$, alter mitochondrial morphology and functionality, and activate endoplasmic reticulum (ER) stress $(8,9,30-32)$, leading to cell type-specific damage. Blocking the access of sUA to the cytoplasm using UA transport inhibitors significantly prevented sUA-induced ROS production and oxidative damage (5-9,12-14). Chondrocytes are characterized by their sensitivity to oxidative stress. ROS in chondrocytes directly activated mitochondrial oxidation (33), ER stress (34) and various signaling pathways, including p38MAPK, ERK1/2 and NF- $\kappa$ B pathways (35), leading to alterations in cell viability and functionality. Thus, it was hypothesized that intracellular sUA may influence chondrocyte viability and functionality. The identification of a UA transport system in chondrocytes is central for research into sUA-induced changes in chondrocytes, and may support future interventions targeted against sUA-induced chondrocyte damage in patients in early stages of gout.

In the present study, GLUT9 and URAT1 were revealed to be active urate transporters in chondrocytes. Previous studies have reported that the two transporters are crucial for regulating UA homeostasis and are subject to various regulatory mechanisms. For example, at the transcriptional level, the GLUT9 promoter contains a conserved response element of STAT5b, which transduces the negative regulatory effects of growth hormone (36). Similarly, the promoter region of 

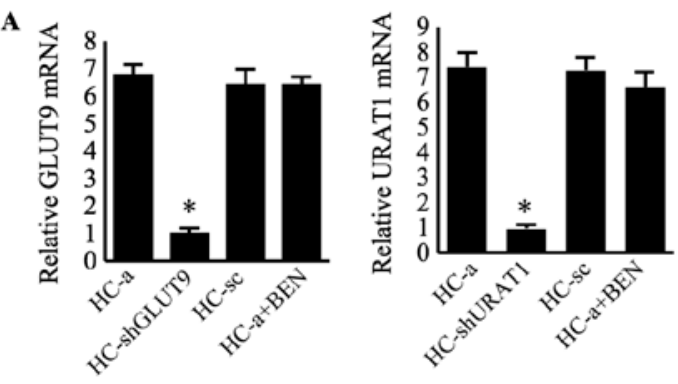

B
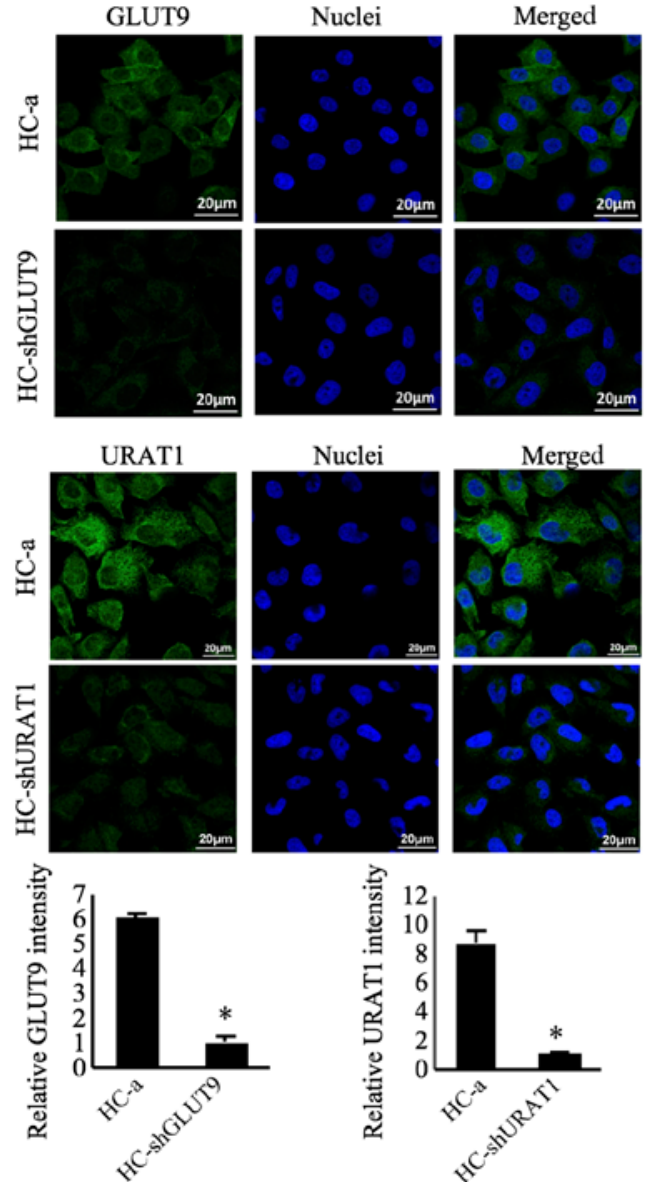

C
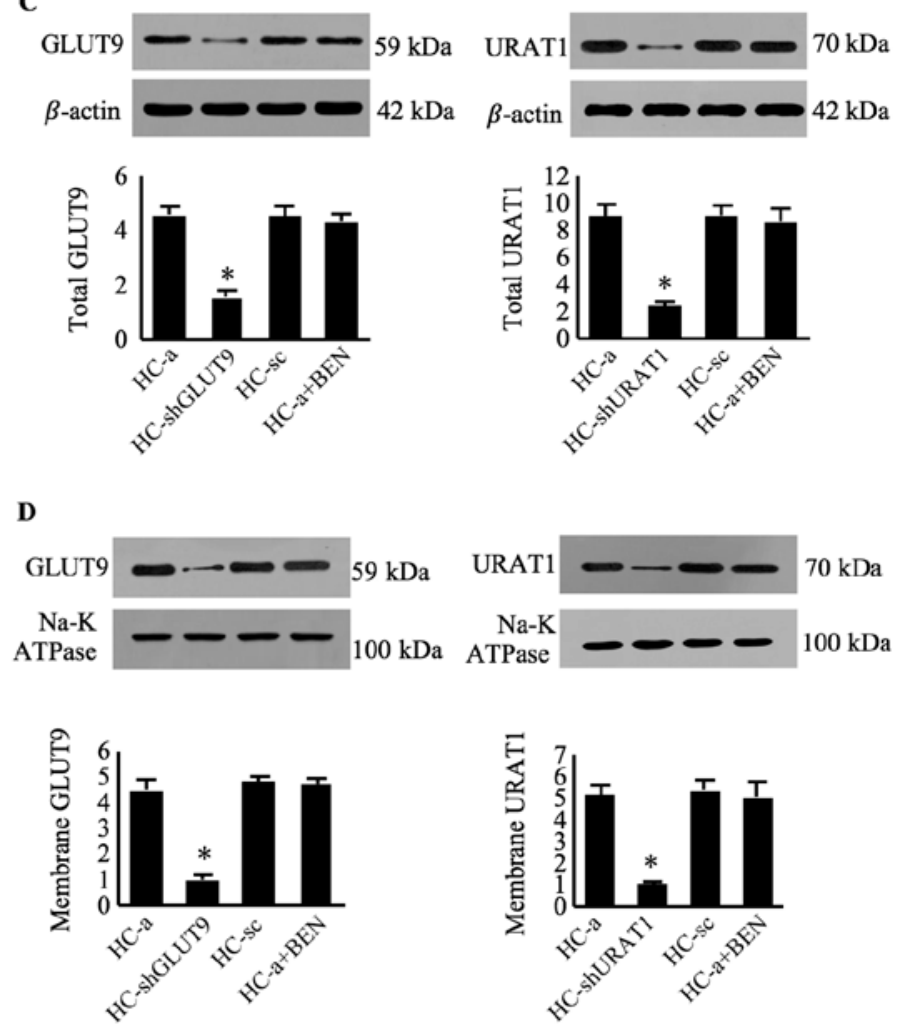

$\mathbf{E}$
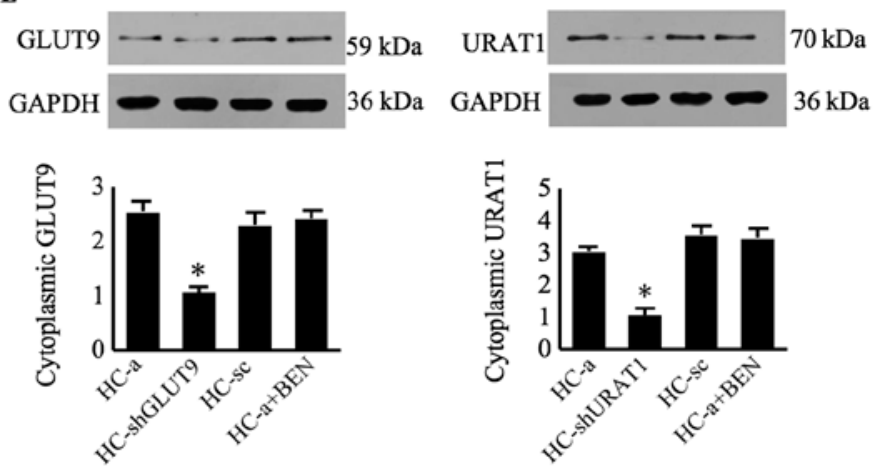

Figure 3. Expression of GLUT9 and URAT1 in human chondrocyte cells. (A) Reverse transcription-quantitative PCR analysis of GLUT9 and URAT mRNA expression in HC-a cells, using $\beta$-actin as an endogenous control. (B) Immunofluorescence of HC-a, HC-shGLUT9 and HC-shURAT1 cells using FITC-conjugated mouse anti-rabbit immunoglobulin $\mathrm{G}$ antibodies (green) and DAPI (blue). Western blot analysis of (C) total protein, (D) membrane protein and (E) cytoplasmic protein content, using $\beta$-actin, Na-K ATPase and GADPH as loading controls, respectively. Data were presented as the mean \pm standard deviation (n=3). * P<0.05 vs. HC-a. GLUT9, glucose transporter 9; URAT1, urate transporter 1; sh, short hairpin RNA; HC-shGLUT9, HC-a cells with GLUT9 knockdown; HC-shURAT1, HC-a cells with URAT1 knockdown; HC-sc, HC-a cells transduced with scrambled RNA; BEN, benzbromarone.

URAT1 contains two GATA sites, which may be responsible for the binding of testosterone (37). In vivo studies with mice confirmed that GLUT9 expression was subject to the inhibitory effects of male-pattern growth hormone secretion (36), whereas URAT1 mRNA was 2.3 times higher in male mice than in female mice (38). In addition, IL- $1 \beta$ stimulation significantly increased the mRNA levels of GLUT9 in chondrocytes (39), whereas tumor suppressor p53 promoted GLUT9 mRNA levels by binding to the promoter region with increased UA transport in fibroblast cell lines and lung cancer cells (40). The promoter region of URAT1 also contains 2 activating protein (AP)-1 sites, 4 AP- 4 sites, 1 hepatocyte nuclear factor 1 site and a CCAAT enhancer binding protein $\beta$ site (37), indicating its potential for regulation. Furthermore, a hyperuricemic mouse model exhibited decreased levels of URAT1 mRNA, whereas obese mice possessed increased levels of URAT1 Mrna (41,42). These results highlight the role of the two transporters in maintaining UA homeostasis during different physiological and pathological conditions. At the post-translational level, the two proteins require targeting to the cell membrane, which may be regulated by endocytic processes. The N-terminal dileucine motif in GLUT9 may be involved in endocytic regulation, as this motif was responsible for the endocytosis of GLUT4, GLUT8 and GLUT12 (43). In 
A

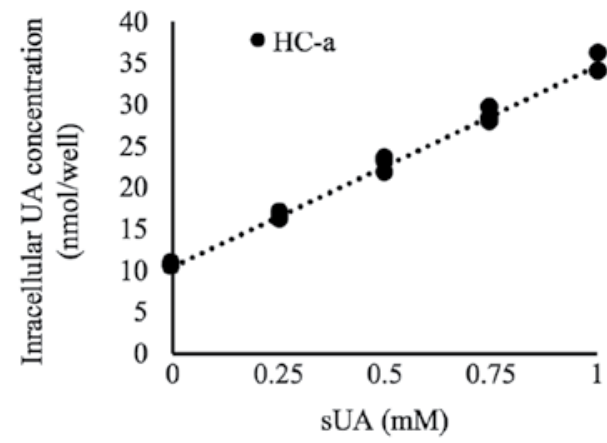

B

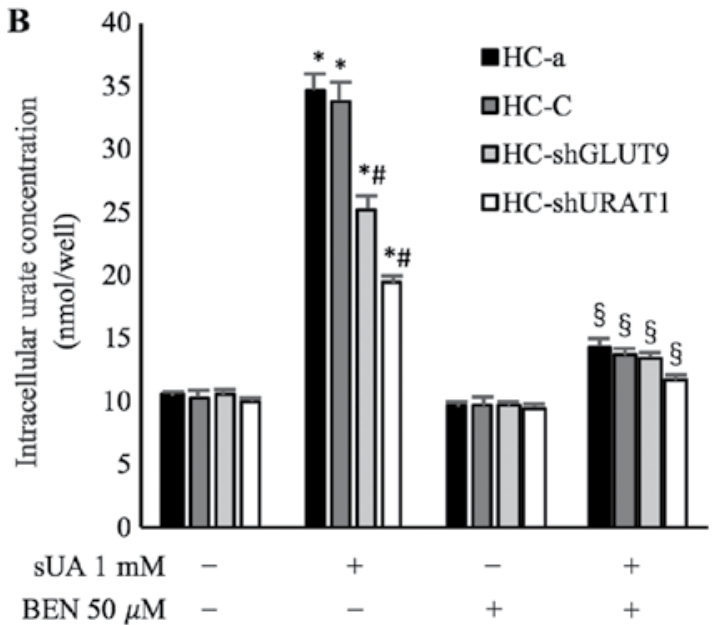

Figure 4. UA transport assay of GLUT9 and URAT1 in chondrocytes. (A) HC-a cells displayed a concentration-dependent increase in intracellular UA concentrations following incubation with exogenous sUA $\left(\mathrm{R}^{2}=0.9932\right)$. (B) Intracellular UA concentrations in cells following incubation with sUA $(1 \mathrm{mM})$ with or without BEN $(50 \mu \mathrm{M})$ pretreatment. Data were presented as the mean \pm standard deviation $(n=3)$. ${ }^{*} \mathrm{P}<0.05$ vs. Control; ${ }^{\#} \mathrm{P}<0.05$ vs. HC-a cells under the same incubation conditions; ${ }^{\circledR} \mathrm{P}<0.05$ vs. sUA. GLUT9, glucose transporter 9; URAT1, urate transporter 1; sh, short hairpin RNA; HC-shGLUT9, HC-a cells with GLUT9 knockdown; HC-shURAT1, HC-a cells with URAT1 knockdown; HC-sc, HC-a cells transduced with scrambled RNA; BEN, benzbromarone; (s)UA, (soluble) uric acid.

293 cells, Wu et al (44) reported that Numb, a clathrin-coated pit adapter protein, bound to URAT1 via its phosphotyrosine-binding domain, facilitating the endocytic regulation of URAT1 under normal and pathological conditions. Due to the importance of plasma membrane density in transporter function, improved understanding of endocytic processes that regulate GLUT9 and URAT1 spatial density may reveal novel trafficking processes that can be targeted to control UA transport in chondrocytes.

At the protein level, the activities of the two proteins are regulated by a number of drugs. Among these, benzbromarone is a strong uricosuric agent that inhibits GLUT9 $\left(\mathrm{IC}_{50}\right.$ $\sim 14.2 \mu \mathrm{mol} / \mathrm{l})$ and URAT1 $\left(\mathrm{IC}_{50} \sim 0.45 \mu \mathrm{mol} / \mathrm{l}\right)$ in irreversible manners $(45,46)$. The mechanism of benzbromarone is considered functional. In GLUT9, the binding pocket is formed by critical transmembrane domain 7 (TM7) and other residues, including N429, C181, C301, C459, C128, I355 and W110 (47,48). Among these residues, C181, the cysteine residue that faces the translocation pore, may be responsible for inhibitor binding and substrate transport function, as proposed by a protein homological study (48). In URAT1,
A
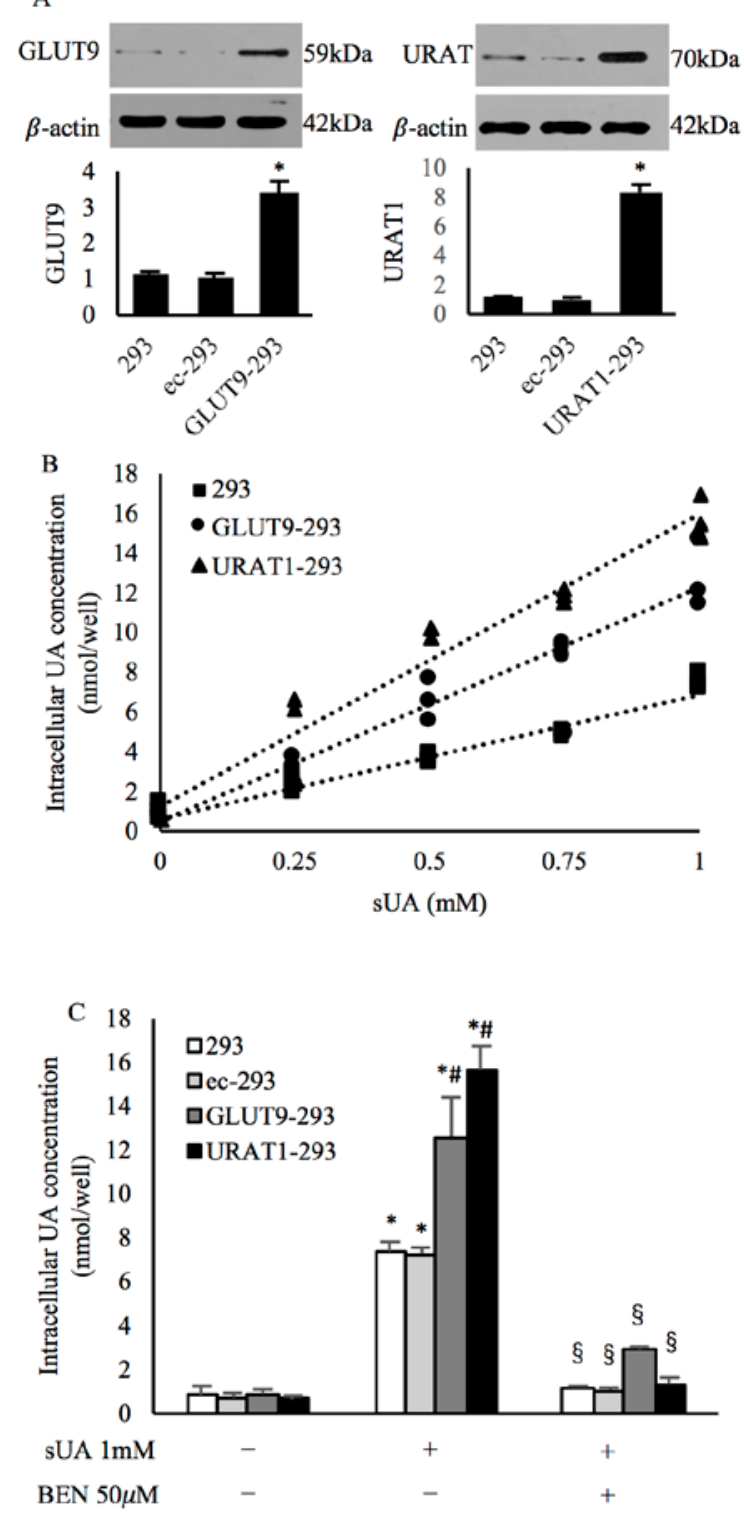

Figure 5. Expression and UA transport assay in 293 cells. (A) Western blot analysis demonstrating overexpression of GLUT9 and URAT1 in transduced 293 cells. ${ }^{*} \mathrm{P}<0.05$ vs. 293. (B) Concentration-dependent increases in intracellular UA concentrations in 293, GLUT9-293 and URAT1-293 cells. (C) Intracellular UA concentrations in cells following incubation with sUA $(1 \mathrm{mM})$ with or without BEN $(50 \mu \mathrm{M})$ pretreatment. Transduction with empty vector did not influence protein expression or transport function. Data were presented as the mean \pm standard deviation $(\mathrm{n}=3)$. ${ }^{*} \mathrm{P}<0.05$ vs. Control; ${ }^{\#} \mathrm{P}<0.05$ vs. HC-a cells under the same incubation conditions; ${ }^{\circledR} \mathrm{P}<0.05$ vs. sUA. GLUT9, glucose transporter 9; URAT1, urate transporter 1; GLUT9-293, 293 cells overexpressing GLUT9; URAT1-293, 293 cells overexpressing URAT1; BEN, benzbromarone; (s)UA, (soluble) uric acid.

3 key residues, F365 on TM7, S35 on TM1 and I481 on TM11, form a plane in the channel that is perpendicular to the cell membrane. A loss-of-function mutation of any of these amino acids resulted in a reduced binding affinity of benzbromarone by 17-, 10-, and 5-fold, respectively. Similarly, F365 and S35 were important for uric acid binding affinity, as mutation of the two amino acids resulted in a 2.5- and 2.1-fold decrease in uric acid binding affinity, respectively. Thus, it was proposed that benzbromarone functioned by preventing substrate interactions within the central portion of the URAT1 channel (45). 
The present findings revealed that neither total nor membrane expression of the two transporters was altered following incubation of HC-a cells with benzbromarone, which supported the notion that the drug was a functional inhibitor without affecting transporter expression or subcellular location.

There were certain limitations to the present study. GLUT9 exists as one of two splicing variants, GLUT9L and GLUT9S, which differ in their N-terminals and cell distribution (49). The antibody used in the present study recognizes the C-terminal of the GLUT9 protein, and therefore could not distinguish between the two variants; however, previous studies have reported two prominent bands in chondrocytes of 60 and $50 \mathrm{kDa}$, suggesting the existence of two different splice variants (50). Furthermore, as benzbromarone also inhibited other minor, facilitative urate transporters, including organic anion transporter (OAT)-1 (Ki 0.22 $\mu \mathrm{M})$ (28), OAT3 (Ki $0.11 \mu \mathrm{M})$ (28), OAT4 $\left(\mathrm{IC}_{50} 3.19 \mu \mathrm{M}\right)(51)$, and OAT10 $\left(\mathrm{IC}_{50} \sim 20 \mu \mathrm{M}\right)(46)$, these OATs may also be components of the UA transport system in chondrocytes. The expression and UA transport capacity of the minor urate transporters, as well as ATP-binding cassette subfamily G member 2, nicotinate phosphoribosyltransferase (NPT)1, NPT4 and multidrug resistance-associated protein 4 may be investigated in future studies to obtain further understanding of the UA transport system in chondrocytes. Additionally, the present study used colorimetric UA transport assays, which are qualitative, but do not allow for the assessment of UA uptake kinetics; however, the results did verify the sensitivity of the colorimetric UA transport assays in positive control 293 cells following GLUT9 and URAT1 overexpression, which was consistent with radiolabeled assays previously employed in this cell line $(14,28)$. Finally, the present study failed to obtain cartilage samples from patients with gout, and to explore the regulation of the two transporters during in vitro experiments, which may provide evidence regarding the pathological regulation of the two transporters. Such regulations, as well as subsequent changes in cell viability and functionality, will be the focus of future studies.

In conclusion, to the best of our knowledge, the present study is the first to report that chondrocytes obtain an active UA transport system mediated by GLUT9 and URAT1, which may be targets for pathological regulation and therapeutic interventions. The involvement of chondrocytes in gouty arthritis may be complex. Further studies into the impact of sUA on chondrocyte ROS production, viability and functionality, and UA transport regulation during normal and pathological conditions, may reveal the potential roles of urate transporters during gouty arthritis. These findings may identify promising targets in UA transport processes for the early control of chronic gouty arthritis.

\section{Acknowledgements}

Not applicable.

\section{Funding}

This study was supported by the 2016 PUMCH Science Fund for Junior Faculty (grant no. pumch-2016-2.13), the Medical
Epigenetics Research Center, Chinese Academy of Medical Sciences (grant no. 2017PT31035), the CAMS Innovation Fund for Medical Sciences (grant no. 2016-I2M-1-002), and the Chinese Research Special Fund for Public Welfare industry of Health (grant no. 201502024).

\section{Availability of data and materials}

The datasets used and/or analyzed during the current study are available from the corresponding author on reasonable request.

\section{Authors' contributions}

$\mathrm{BiZ}$ and MD designed the study and drafted the manuscript. BiZ, MD and BL conducted the majority of the experiments. BaZ and YJ enrolled the patients and collected the tissue samples. BiZ, DW and YZ analyzed the data. JC and $\mathrm{XH}$ interpreted the data and revised the manuscript for critical intellectual content. LZ and XZ contributed to the study design, provided assistance with the experiments and revised the manuscript. All authors read and approved the manuscript.

\section{Ethics approval and consent to participate}

This study was approved by the institutional review board of Peking Union Medical College Hospital (permit no. ZS-1445) and was conducted in accordance with the Declaration of Helsinki (2000) of the World Medical Association. All patients signed informed consent prior to tissue sample collection.

\section{Patient consent for publication}

Not applicable.

\section{Competing interests}

The authors declare that they have no competing interests.

\section{References}

1. Chhana A, Callon KE, Pool B, Naot D, Gamble GD, Dray M, Pitto R, Bentley J, McQueen FM, Cornish J and Dalbeth N: The effects of monosodium urate monohydrate crystals on chondrocyte viability and function: Implications for development of cartilage damage in gout. J Rheumatol 40: 2067-2074, 2013.

2. Liu R, Lioté F, Rose DM, Merz D and Terkeltaub R: Proline-rich tyrosine kinase 2 and Src kinase signaling transduce monosodium urate crystal-induced nitric oxide production and matrix metalloproteinase 3 expression in chondrocytes. Arthritis Rheum 50: 247-258, 2004.

3. Hwang HS, Yang CM, Park SJ and Kim HA: Monosodium urate crystal-induced chondrocyte death via autophagic process. Int J Mol Sci 16: 29265-29277, 2015.

4. Martillo MA, Nazzal L and Crittenden DB: The crystallization of monosodium urate. Curr Rheumatol Rep 16: 400, 2014.

5. Kang DH: Hyperuricemia and progression of chronic kidney disease: Role of phenotype transition of renal tubular and endothelial cells. Contrib Nephrol 192: 48-55, 2018.

6. Liang WY, Zhu XY, Zhang JW, Feng XR, Wang YC and Liu ML: Uric acid promotes chemokine and adhesion molecule production in vascular endothelium via nuclear factor-kappa B signaling. Nutr Metab Cardiovasc Dis 25: 187-194, 2015 
7. Kang DH, Han L, Ouyang X, Kahn AM, Kanellis J, Li P, Feng L, Nakagawa T, Watanabe S, Hosoyamada M, et al: Uric acid causes vascular smooth muscle cell proliferation by entering cells via a functional urate transporter. Am J Nephrol 25: 425-433, 2005.

8. Kırça M, Oğuz N, Çetin A, Uzuner F and Yeşilkaya A: Uric acid stimulates proliferative pathways in vascular smooth muscle cells through the activation of p38 MAPK, p44/42 MAPK and PDGFR $\beta$. J Recept Signal Transduct Res 37: 167-173, 2017

9. Choi YJ, Shin HS, Choi HS, Park JW, Jo I, Oh ES, Lee KY, Lee BH, Johnson RJ and Kang DH: Uric acid induces fat accumulation via generation of endoplasmic reticulum stress and SREBP-1c activation in hepatocytes. Lab Invest 94: 1114-1125, 2014.

10. Xin Y, Wang K, Jia Z, Xu T, Xu Q, Zhang C, Liu J, Chen R, Du Z and Sun J: Zurampic protects pancreatic beta-cells from high uric acid induced-damage by inhibiting URAT1 and inactivating the ROS/AMPK/ERK pathways. Cell Physiol Biochem 47: 1074-1083, 2018.

11. Zhang Y, Yamamoto T, Hisatome I, Li Y, Cheng W, Sun N, Cai B, Huang T, Zhu Y, Li Z, et al: Uric acid induces oxidative stress and growth inhibition by activating adenosine monophosphateactivated protein kinase and extracellular signal-regulated kinase signal pathways in pancreatic $\beta$ cells. Mol Cell Endocrinol 375 : 89-96, 2013

12. Sautin YY, Nakagawa T, Zharikov S and Johnson RJ: Adverse effects of the classic antioxidant uric acid in adipocytes: NADPH oxidase-mediated oxidative/nitrosative stress. Am J Physiol Cell Physiol 293: C584-C596, 2007.

13. Evans SA, Doblado M, Chi MM, Corbett JA and Moley KH: Facilitative glucose transporter 9 expression affects glucose sensing in pancreatic beta-cells. Endocrinology 150: 5302-5310, 2009.

14. Caulfield MJ, Munroe PB, O'Neill D, Witkowska K, Charchar FJ, Doblado M, Evans S, Eyheramendy S, Onipinla A, Howard P, et al: SLC2A9 is a high-capacity urate transporter in humans. PLoS Med 5:e197, 2008.

15. Nigam SK and Bhatnagar V: The systems biology of uric acid transporters: The role of remote sensing and signaling. Curr Opin Nephrol Hypertens 27: 305-313, 2018.

16. Preitner F, Bonny O, Laverriere A, Rotman S, Firsov D, Da Costa A, Metref S and Thorens B: Glut9 is a major regulator of urate homeostasis and its genetic inactivation induces hyperuricosuria and urate nephropathy. Proc Natl Acad Sci USA 106 15501-15506, 2009.

17. Tu HP, Chen CJ, Tovosia S, Ko AM, Lee CH, Ou TT, Lin GT, Chang SJ, Chiang SL, Chiang HC, et al: Associations of a non-synonymous variant in SLC2A9 with gouty arthritis and uric acid levels in Han Chinese subjects and solomon islanders. Ann Rheum Dis 69: 887-890, 2010.

18. Nakanishi T, Ohya K, Shimada S, Anzai N and Tamai I: Functional cooperation of URAT1 (SLC22A12) and URATv1 (SLC2A9) in renal reabsorption of urate. Nephrol Dial Transplant 28: 603-611, 2013.

19. Price KL, Sautin YY, Long DA, Zhang L, Miyazaki H, Mu W, Endou H and Johnson RJ: Human vascular smooth muscle cells express a urate transporter. J Am Soc Nephrol 17: 1791-1795, 2006.

20. Richardson S, Neama G, Phillips T, Bell S, Carter SD, Moley KH, Moley JF, Vannucci SJ and Mobasheri A: Molecular characterization and partial cDNA cloning of facilitative glucose transporters expressed in human articular chondrocytes; stimulation of 2-deoxyglucose uptake by IGF-I and elevated MMP-2 secretion by glucose deprivation. Osteoarthritis Cartilage 11 : 92-101, 2003

21. Zhai K, Tang Y, Zhang Y, Li F, Wang Y, Cao Z, Yu J, Kou J and Yu B: NMMHC IIA inhibition impedes tissue factor expression and venous thrombosis via Akt/GSK3beta-NF-kappaB signalling pathways in the endothelium. Thromb Haemost 114: $173-185,2015$.

22. Zhai KF, Zheng JR, Tang YM, Li F, Lv YN, Zhang YY, Gao Z, Qi J, Yu BY and Kou JP: The saponin D39 blocks dissociation of non-muscular myosin heavy chain IIA from TNF receptor 2, suppressing tissue factor expression and venous thrombosis. $\mathrm{Br}$ J Pharmacol 174: 2818-2831, 2017.

23. Livak KJ and Schmittgen TD: Analysis of relative gene expression data using real-time quantitative PCR and the 2(-Delta Delta C(T)) method. Methods 25: 402-408, 2001.

24. Zhai KF, Duan H, Khan GJ, Xu H, Han FK, Cao WG, Gao GZ, Shan LL and Wei ZJ: Salicin from alangium chinense ameliorates rheumatoid arthritis by modulating the Nrf2-HO-1-ROS pathways. J Agric Food Chem 66: 6073-6082, 2018.
25. Anzai N, Ichida K, Jutabha P, Kimura T, Babu E, Jin CJ, Srivastava S, Kitamura K, Hisatome I, Endou H and Sakurai H: Plasma urate level is directly regulated by a voltage-driven urate efflux transporter URATv1 (SLC2A9) in humans. J Biol Chem 283: 26834-26838, 2008.

26. Enomoto A, Kimura H, Chairoungdua A, Shigeta Y, Jutabha P, Cha SH, Hosoyamada M, Takeda M, Sekine T, Igarashi T, et al: Molecular identification of a renal urate anion exchanger that regulates blood urate levels. Nature 417: 447-452, 2002.

27. Eyre D: Collagen of articular cartilage. Arthritis Res 4: 30-35, 2002.

28. Ahn SO, Ohtomo S, Kiyokawa J, Nakagawa T, Yamane M, Lee KJ, Kim KH, Kim BH, Tanaka J, Kawabe Y and Horiba N: Stronger uricosuric effects of the novel selective URAT1 inhibitor UR-1102 lowered plasma urate in tufted capuchin monkeys to a greater extent than benzbromarone. J Pharmacol Exp Ther 357: 157-166, 2016.

29. Sharaf El Din UAA, Salem MM and Abdulazim DO: Uric acid in the pathogenesis of metabolic, renal, and cardiovascular diseases: A review. J Adv Res 8: 537-548, 2017.

30. Kanellis J, Watanabe S, Li JH, Kang DH, Li P, Nakagawa T, Wamsley A, Sheikh-Hamad D, Lan HY, Feng L and Johnson RJ: Uric acid stimulates monocyte chemoattractant protein-1 production in vascular smooth muscle cells via mitogen-activated protein kinase and cyclooxygenase-2. Hypertension 41: 1287-1293, 2003.

31. Jia L, Xing J, Ding Y, Shen Y, Shi X, Ren W, Wan M, Guo J, Zheng S, Liu Y, et al: Hyperuricemia causes pancreatic beta-cell death and dysfunction through NF-kappaB signaling pathway. PLoS One 8: e78284, 2013.

32. Corry DB, Eslami P, Yamamoto K, Nyby MD, Makino H and Tuck ML: Uric acid stimulates vascular smooth muscle cell proliferation and oxidative stress via the vascular renin-angiotensin system. J Hypertens 26: 269-275, 2008.

33. Wu L, Liu H, Li L, Liu H, Cheng Q, Li H and Huang H: Mitochondrial pathology in osteoarthritic chondrocytes. Curr Drug Targets 15: 710-719, 2014.

34. Takada K, Hirose J, Yamabe S, Uehara Y and Mizuta H: Endoplasmic reticulum stress mediates nitric oxide-induced chondrocyte apoptosis. Biomed Rep 1: 315-319, 2013.

35. Henrotin YE, Bruckner P and Pujol JP: The role of reactive oxygen species in homeostasis and degradation of cartilage. Osteoarthritis Cartilage 11: 747-755, 2003.

36. Bu P, Le Y, Zhang Y and Cheng X: Hormonal and chemical regulation of the Glut9 transporter in Mice. J Pharmacol Exp Ther 360: 206-214, 2017.

37. Li T, Walsh JR, Ghishan FK and Bai L: Molecular cloning and characterization of a human urate transporter (hURAT1) gene promoter. Biochim Biophys Acta 24: 53-58, 2004.

38. Hosoyamada M, Ichida K, Enomoto A, Hosoya T and Endo H: Function and localization of urate transporter 1 in mouse kidney. J Am Soc Nephrol 15: 261-268, 2004.

39. Shikhman AR, Brinson DC, Valbracht J and Lotz MK: Cytokine regulation of facilitated glucose transport in human articular chondrocytes. J Immunol 15: 7001-7008, 2001

40. Itahana Y, Han R, Barbier S, Lei Z, Rozen S and Itahana K: The uric acid transporter SLC2A9 is a direct target gene of the tumor suppressor 533 contributing to antioxidant defense. Oncogene 34 : $1799-1810,2015$.

41. Nishizawa K, Yoda N, Morokado F, Komori H, Nakanishi T and Tamai I: Changes of drug pharmacokinetics mediated by downregulation of kidney organic cation transporters Matel and Oct2 in a rat model of hyperuricemia. PLoS One 14: e0214862, 2019.

42. Doshi M, Takiue Y, Saito $\mathrm{H}$ and Hosoyamada $\mathrm{M}$ : The increased protein level of URAT1 was observed in obesity/metabolic syndrome model mice. Nucleosides Nucleotides Nucleic Acids 30: 1290-1294, 2011

43. Augustin R, Carayannopoulos MO, Dowd LO, Phay JE, Moley JF and Moley KH: Identification and characterization of human glucose transporter-like protein-9 (GLUT9): Alternative splicing alters trafficking. J Biol Chem 279: 16229-16236, 2004.

44. Wu X, Zhang J, Liu T, Yan M, Liu H, Xie H, Zhang S, Sun B, $\mathrm{Ke} \mathrm{B}$ and Zhou $\mathrm{H}$ : Uric acid crystal could inhibit numb-induced URAT1 lysosome degradation in uric acid nephropathy. J Physiol Biochem 17: 217-226, 2015.

45. Tan PK, Ostertag TM and Miner JN: Mechanism of high affinity inhibition of the human urate transporter URAT1. Sci Rep 6: 34995, 2016.

46. Mandal AK, Mercado A, Foster A, Zandi-Nejad K and Mount DB: Uricosuric targets of tranilast. Pharmacol Res Perspect 5: e00291, 2017. 
47. Long W, Panwar P, Witkowska K, Wong K, O'Neill D, Chen XZ, Lemieux MJ and Cheeseman CI: Critical roles of two hydrophobic residues within human glucose transporter 9 (hSLC2A9) in substrate selectivity and urate transport. J Biol Chem 290: 15292-15303, 2015.

48. Long W, Panigrahi R, Panwar P, Wong K, O Neill D, Chen XZ, Lemieux MJ and Cheeseman CI: Identification of key residues for urate specific transport in human glucose transporter 9 (hSLC2A9). Sci Rep 7: 41167, 2017.

49. Kimura T, Takahashi M, Yan K and Sakurai H: Expression of the GLUT1 and GLUT9 facilitative glucose transporters in embryonic chondroblasts and mature chondrocytes in ovine articular cartilage. PLoS One 9: e84996, 2014.

50. Mobasheri A, Dobson H, Mason SL, Cullingham F, Shakibaei M, Moley JF and Moley KH: Expression of the GLUT1 and GLUT9 facilitative glucose transporters in embryonic chondroblasts and mature chondrocytes in ovine articular cartilage. Cell Biol Int 29: 249-260, 2005.
51. Miner J, Tan PK, Hyndman D, Liu S, Iverson C, Nanavati P, Hagerty DT, Manhard K, Shen Z, Girardet JL, et al: Lesinurad, a novel, oral compound for gout, acts to decrease serum uric acid through inhibition of urate transporters in the kidney. Arthritis Res Ther 18: 214, 2016.

(i) (9) This work is licensed under a Creative Commons Attribution-NonCommercial-NoDerivatives 4.0 International (CC BY-NC-ND 4.0) License. 